\title{
Kley, Thomas
}

\section{Die Migrationssozialarbeit der Caritas}

Spetsmann-Kunkel, Martin [Hrsg.]; Frieters-Reermann, Norbert [Hrsg.]: Soziale Arbeit in der Migrationsgesellschaft. Opladen ; Berlin ; Toronto : Verlag Barbara Budrich 2013, S. 147-150. - (Schriften der Katholischen Hochschule Nordrhein-Westfalen; 17)

Quellenangabe/ Reference:

Kley, Thomas: Die Migrationssozialarbeit der Caritas - In: Spetsmann-Kunkel, Martin [Hrsg.];

Frieters-Reermann, Norbert [Hrsg.]: Soziale Arbeit in der Migrationsgesellschaft. Opladen ; Berlin ;

Toronto : Verlag Barbara Budrich 2013, S. 147-150 - URN: urn:nbn:de:0111-pedocs-127903 - DOI: $10.25656 / 01: 12790$

https://nbn-resolving.org/urn:nbn:de:0111-pedocs-127903

https://doi.org/10.25656/01:12790

in Kooperation mit / in cooperation with:

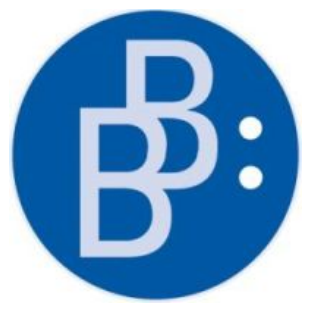

https://www.budrich.de

\section{Nutzungsbedingungen}

Dieses Dokument steht unter folgender Creative Commons-Lizenz:

$\mathrm{http}: / /$ creativecommons.org/licenses/by-nc-nd/3.0/de/deed - Sie dürfen das Werk bzw. den Inhalt unter folgenden Bedingungen vervielfältigen, verbreiten und öffentlich zugänglich machen: Sie müssen den Namen des Autors/Rechteinhabers in der von ihm festgelegten Weise nennen. Dieses Werk bzw. dieser Inhalt darf nicht für kommerzielle Zwecke verwendet werden und es darf nicht bearbeitet, abgewandelt oder in anderer Weise verändert werden.

Mit der Verwendung dieses Dokuments erkennen Sie die Nutzungsbedingungen an.

\section{Terms of use}

This document is published under following Creative Commons-License: http://creativecommons.org/licenses/by-nc-nd/3.0/de/deed.en - You may copy, distribute and transmit, adapt or exhibit the work in the public as long as you attribute the work in the manner specified by the author or licensor. You are not allowed to make commercial use of the work or its contents. You are not allowed to alter, transform, or change this work in any other way.

By using this particular document, you accept the above-stated conditions of use.

\section{Kontakt / Contact:}

peDOcs

DIPF | Leibniz-Institut für Bildungsforschung und Bildungsinformation Informationszentrum (IZ) Bildung

E-Mail: pedocs@dipf.de

Internet: www.pedocs.de

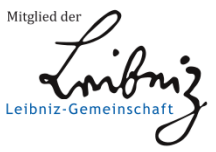


Martin Spetsmann-Kunkel

Norbert Frieters-Reermann (Hrsg.)

\section{Soziale Arbeit in der Migrationsgesellschaft}

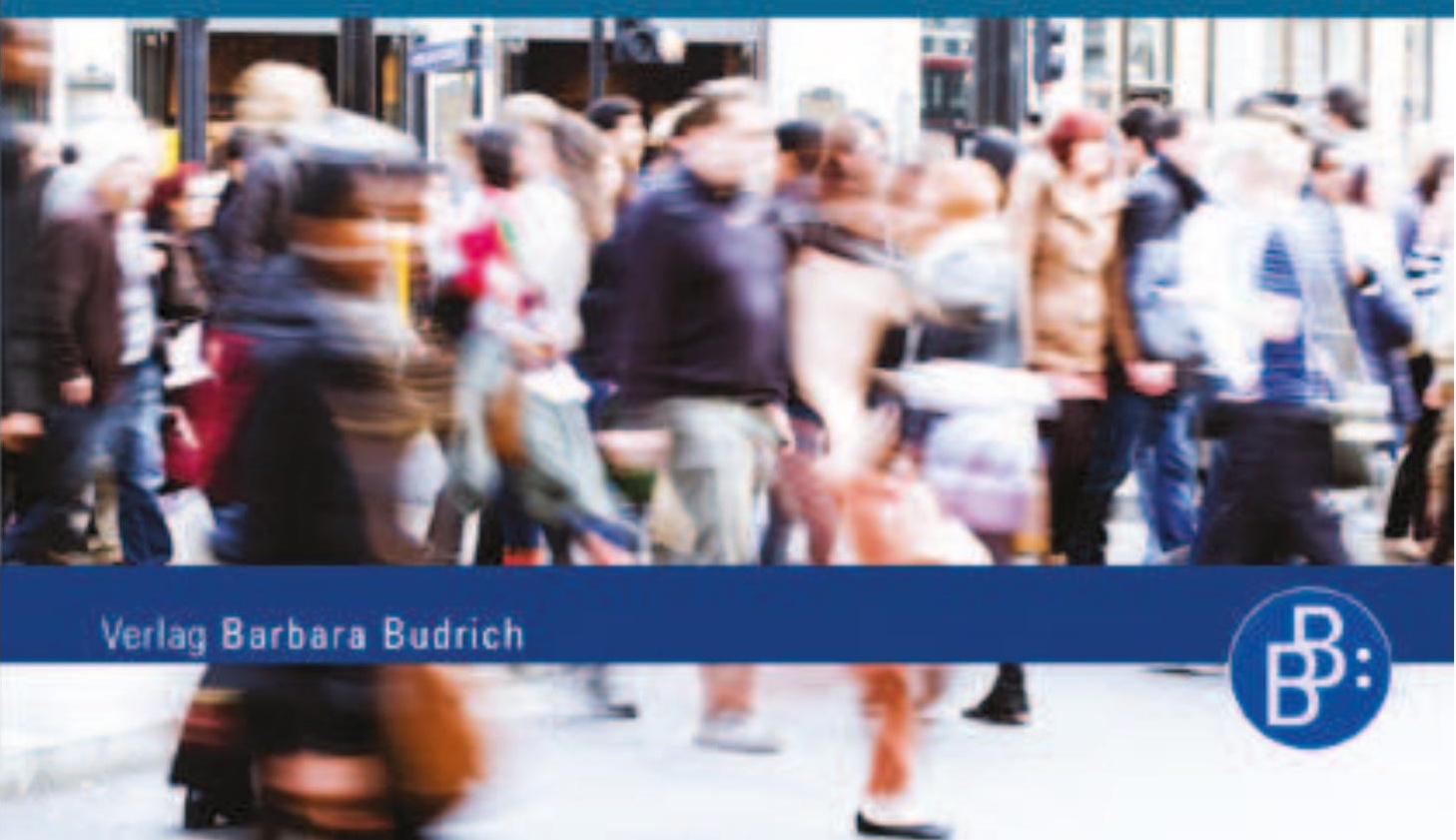


Schriften der Katholischen Hochschule

Nordrhein-Westfalen

Band 17

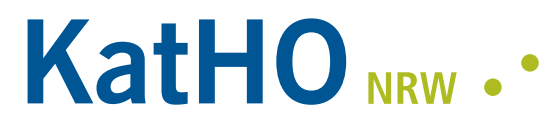

Aachen | Köln | Münster | Paderborn

Katholische Hochschule Nordrhein-Westfalen Catholic University of Applied Sciences 
Martin Spetsmann-Kunkel

Norbert Frieters-Reermann (Hrsg.)

\section{Soziale Arbeit in der} Migrationsgesellschaft

Verlag Barbara Budrich

Opladen, Berlin \& Toronto 2013 
Prof. Dr. Martin Spetsmann-Kunkel

Prof. Dr. Norbert Frieters-Reermann

Katholische Hochschule NRW / Aachen

Robert-Schuman-Straße 25

52066 Aachen

Tel.: (+49) (0)241-6000318

Bibliografische Information der Deutschen Nationalbibliothek

Die Deutsche Nationalbibliothek verzeichnet diese Publikation in der Deutschen

Nationalbibliografie; detaillierte bibliografische Daten sind im Internet über

http://dnb.d-nb.de abrufbar.

(C) 2013 Dieses Werk ist im Verlag Barbara Budrich erschienen und steht unter folgender Creative Commons Lizenz: http://creativecommons.org/licenses/by-nc$\mathrm{nd} / 3.0 / \mathrm{de} /$

Verbreitung, Speicherung und Vervielfältigung erlaubt, kommerzielle Nutzung und Veränderung nur mit Genehmigung des Verlags Barbara Budrich

Dieses Buch steht im OpenAccess Bereich der Verlagsseite zum kostenlosen Download bereit (https://doi.org/10.3224/93809468)

Eine kostenpflichtige Druckversion kann über den Verlag bezogen werden. Die Seitenzahlen in der Druck- und Onlineversion sind identisch.

\section{ISBN 978-3-938094-68-6 (Paperback) \\ eISBN 978-3-8474-0337-1 (eBook) \\ DOI $\quad 10.3224 / 93809468$}

Umschlaggestaltung: disegno visuelle kommunikation, Wuppertal - www.disenjo.de Typografisches Lektorat: Petra Reiners, Bonn - www.buchfinken.com Druck: paper \& tinta, Warschau

Printed in Germany 


\section{Inhaltsverzeichnis}

1. Soziale Arbeit in der Migrationsgesellschaft einleitende Gedanken

Martin Spetsmann-Kunkel

2. Anfragen an die Praxis der Sozialen Arbeit in einer von Mobilität und Vielfalt geprägten Postmoderne - Soziale Arbeit in der Migrationsgesellschaft

Wolf-D. Bukow

3. Interkulturelle Soziale Arbeit: Integration, Anerkennung und Partizipation als Leitideen einer differenzsensiblen Sozialen Arbeit in der Migrationsgesellschaft

Josef Freise

4. Interkulturelles Lernen und Forschen in transkulturellen Dialoggruppen

Cornelia Muth

5. Demokratiepädagogik und Diversity Education pädagogische Konzepte und ihre Bedeutung für die Soziale Arbeit

Schahrzad Farrokhzad 
6. Kritische Soziale Arbeit in Diskriminierungs- und Herrschaftsverhältnissen - eine Skizze

Claus Melter

7. Soziale Arbeit in mobiler Gesellschaft Institutionelle Bedingungen, professionelle Expertise und das eigene Verschränkt-Sein in Alltagsrassismus und kulturalisierenden Deutungsprozessen

Claudia Roller

8. Interkulturelle Öffnung als Team - von Stolpersteinen und Meilensteinen

Norbert Teutenberg

9. Die Migrationssozialarbeit der Caritas

Thomas Kley

10. Soziale Arbeit und Migration - Auslassungen, Anregungen und Ausblicke

Norbert Frieters-Reermann 


\title{
9. Die Migrationssozialarbeit der Caritas
}

\author{
Thomas Kley
}

Für die Caritas ist Solidarität mit Fremden und die Begegnung mit anderen Kulturen Bestandteil des christlichen Selbstverständnisses. Die Caritas bejaht die kulturelle, religiöse und gesellschaftliche Vielfalt und will unsere Vielfaltsgesellschaft als wertegebundener Verband mitgestalten.

Die Caritas im Bistum Aachen unterstützt mit regionalen Fachdiensten für Integration und Migration (FIM) die soziale, politische und gesellschaftliche Teilhabe von Zuwanderern. Die Fachdienste für Integration und Migration (FIM) beraten Zuwanderer individuell bei allen Fragen rund um die persönliche Integration. Gleichzeitig ist es Ziel und Aufgabe, die Integration und Teilhabe von Zuwanderern strukturell zu fördern.

Die regionalen Fachdienste sind besonders Ansprechpartner bei Fragen der interkulturellen Öffnung, der sozialraumorientierten Integrationsarbeit, beim Dialog der Kulturen und Religionen, bei der Förderung des bürgerschaftlichen Engagements von und für Zuwanderer und bei der Antidiskriminierungsarbeit. Die Fachdienste bieten je nach inhaltlicher Ausrichtung verschiedene Beratungsangebote an:

- Die Migrationserstberatung (MBE) richtet sich an erwachsene Zuwanderer, besonders an Neuzuwanderer.

- Der Jugendmigrationsdienst (JMD) richtet sich an junge Zuwanderer, besonders an Neuzuwanderer.

- Die Integrationsagentur (IA) arbeitet an Strukturen der Integrationsförderung im Stadtteil, unterstützt Migrantenorganisationen und berät soziale Einrichtungen und Institutionen bei ihrer interkulturellen Öffnung.

- Die Flüchtlingsberatung richtet sich an Flüchtlinge, unabhängig von ihrem Aufenthaltsstatus.

Der Diözesancaritasverband im Bistum Aachen leistet die spitzenverbandliche Vertretung in Politik und Gesellschaft, informiert die regionalen Fachdienste mit ihren Trägern über migrationspolitische Entwicklungen und berät bei fachlichen und strukturellen Fragen. 


\section{Gesellschaftliche und politische Rahmenbedingungen der Migrationsgesellschaft - eine Bilanz}

Faktisch ist Deutschland im Jahr 2012 ein demografisch alterndes Migrationsland, in NRW haben mehr als 23\% der Bevölkerung einen Migrationshintergrund. Die deutsche Politik war trotzdem jahrzehntelang von der Vorstellung bestimmt, Deutschland sei kein Einwanderungsland und man brauche auch keine Integrationsförderung. Unabhängig von dieser Bewertung hat Einwanderung/Zuwanderung nach Deutschland in einem beträchtlichen Umfang stattgefunden, viele der Zuwanderer sind geblieben und mittlerweile eingebürgert bzw. waren deutschstämmige Aussiedler. Diese (ungesteuerte) Zuwanderung nach Deutschland hat die Überalterung und Schrumpfung der Gesellschaft nicht stoppen, wohl aber zahlenmäßig verringern können. Seit der Anwerbung von Arbeitsmigranten für niedrigqualifizierte Arbeitsplätze ab den 1950er Jahren wurde versäumt, bestehende sprachliche und soziale Nachteile auszugleichen. Das hat sich auch bei den nachfolgenden Generationen dieser Arbeitsmigranten durch eine systematische Bildungsbenachteiligung fortgesetzt und zur sozioökonomischen „Unterschichtung“ unserer Gesellschaft beigetragen.

Auf Bundesebene gab es erst 2001 mit dem Bericht der unabhängigen „Süssmuth-Kommission“ einen Perspektivwechsel in der Ausgestaltung der Integrationspolitik. In der Folge wurden dann mehrere Gesetze verabschiedet, wobei das Zuwanderungsgesetz von 2005 das bekannteste ist. Der Bund will mit seiner Gesetzgebung stärker als bisher seine gestaltende Verantwortung bei Angeboten zur Integrationsförderung deutlich machen.

Das Bundesland Nordrhein-Westfalen (NRW) hatte für den Bund und andere Bundesländer eine Vorbild-Funktion bei der Integrationspolitik und für den Umgang mit Fragen von Zuwanderung und gesellschaftlicher Vielfalt. Kennzeichnend dafür sind die parteiübergreifende Integrationsoffensive des Landtages NRW aus dem Jahr 2001 und die Schaffung des bundesweit ersten Integrationsministeriums in einem FlächenBundesland. Seit Februar 2012 werden in NRW mit dem „Teilhabe- und IntegrationsGesetz" die Kommunen in ihrer Verantwortung für die Integration von Zuwanderern vor Ort gestärkt. Kommunale Integrationsarbeit wird strukturell gefördert, aber auch als Pflicht verstanden. 


\section{Das Verhältnis von Caritas als Teil der Freien Wohlfahrtspflege und Politik}

Migrationssozialarbeit ist Teil der Sozialpolitik, die bislang in Deutschland auf allen Ebenen von dem Prinzip der Subsidiarität und Pluralität geprägt ist. Der Staat überträgt freien Trägern wie den Wohlfahrtsverbänden bestimmte soziale Aufgaben und finanziert diese anteilig. Der Dialog zwischen Politiker/innen und Vertreter/innen der Wohlfahrtsverbände basiert noch auf diesem gemeinsamen Verständnis. Allerdings ist zu beobachten, dass die Erfahrungen und die fachlichen Kompetenzen von Wohlfahrtsverbänden als einer wichtigen gesellschaftlichen Gruppe weniger als vor ein bis zwei Jahrzehnten bei politischen Entscheidungsprozessen einfließen. Diese Entwicklung ist unabhängig von einer parteipolitischen Nähe der verschiedenen Wohlfahrtsverbände und mag einen sozialpolitischen Bedeutungsverlust der Freien Wohlfahrtspflege anzeigen.

Auch hat sich mehrheitlich in der Politik das grundsätzliche Verständnis von Sozialpolitik und Wohlfahrtsstaat massiv verändert. Stärker als bisher wird dem Einzelnen einseitig die Verantwortung für seine soziale Lage und Vorsorge übertragen und dabei $\mathrm{zu}$ wenig auf Benachteiligungen und notwendige Förderung geachtet. Soziale (Einzelfall-)Arbeit für Menschen, die Rat suchen, wird nicht mehr refinanziert, die Bedeutung von Strukturen und Einrichtungen wird überbetont. Das betrifft alle Menschen, unabhängig von ihrer Herkunft.

\section{Fachliche Entwicklungen der Migrationssozialarbeit der Caritas}

Die Migrationssozialarbeit der verbandlichen Caritas im Bistum Aachen ist eingebunden in verbandliche und fachliche Strukturen auf Landes- und Bundesebene. Mit dem Zuwanderungsgesetz von 2005 wurde erstmals eine klare Trennung zwischen der Aufgabe des Bundes für die individuelle Integrationsförderung von Neuzuwanderern und für die Aufgabe der Bundesländer für die strukturelle Integrationsförderung der bereits hier lebenden Zuwanderer definiert. In NRW hat die Caritas alle bisher getrennt arbeitenden Fachdienste (für erwachsene (Erst-)Zuwanderer, für junge (Erst-) Zuwanderer und für Flüchtlinge) bei regionalen Trägern zusammengeführt. Mit dem Land NRW und dem zuständigen Ministerium wurde gleichzeitig das Konzept der Integrationsagenturen umgesetzt. 
Die Fachdienste für Integration und Migration haben verschiedene Aufgaben mit unterschiedlichen Zielgruppen:

1. Individuelle Förderung und Beratung für Neuzuwanderer, Flüchtlinge und Menschen in aufenthaltsrechtlicher Illegalität

2. Aktivierung/Begleitung von interkulturellen Integrationsprozessen bei sozialen Diensten, Einrichtungen, Institutionen, Migrantenorganisationen und im kirchlichen Raum

3. Förderung von Vielfalts-Akzeptanz und Vielfalts-Kompetenz bei Deutschen ohne und mit Migrationshintergrund und in allen gesellschaftlichen Bereichen.

Menschen mit Migrationshintergrund dürfen und können nicht alleine auf die Kategorie „Migrationshintergrund“ reduziert werden. Sie sind genauso z.B. Eltern, Kinder, Arbeitnehmer, Bewohner im Stadtteil, Kranke, Ratsuchende und haben - genauso wie deutsche Einheimische - vergleichbare individuelle Sorgen und Nöte. Entscheidend ist, wie Einrichtungen und soziale Dienste bei Zuwanderern auf deren kulturelle Unterschiedlichkeit und Prägung eingehen können. Integration/Migration als Arbeitsfeld ist eine Querschnittsaufgabe und berührt alle sozialen Arbeitsfelder. Die Fachdienste unterstützen und begleiten die interkulturelle Öffnung der sozialen Regelversorgung, damit Zuwanderer diese entsprechend in Anspruch nehmen können.

\section{Die Migrationssozialarbeit der Caritas als wertegebundener Verband in der Vielfaltsgesellschaft}

Den Anspruch, den die Caritas an die interkulturelle Öffnung der sozialen Regelversorgung stellt, will sie auch selbst erfüllen. Die konsequente interkulturelle Ausrichtung aller Dienste und Einrichtungen der Caritas ist eine der zentralen Herausforderungen. Dabei kommt den Fachdiensten für Integration und Migration die Rolle der fachlichen Begleitung und des „Mahners“ zu. Ein besonderes Thema mit Blick auf Zuwanderer ist für die Caritas die Bindung an die kirchliche Grundordnung. Hier geht es vor allem um die Einstellung und Beschäftigung von muslimischen Mitarbeiter/innen in beratend tätigen Berufsgruppen. Die Fachdienste für Integration und Migration werben in der Caritas für eine weniger enge Auslegung und Anwendung der Grundordnung. Ein interner interreligiöser Dialog zwischen Mitarbeitenden bietet große Chancen zur Glaubensvertiefung des/der Einzelnen und zur Profilschärfung als katholischer Verband. 